\title{
Prévention de la cataracte radio-induite : abaissement de la dose limite réglementaire et évaluation d'activités de maintenance potentiellement à risque en centrale nucléaire
}

\author{
R. D’Aloia' ${ }^{1,2, \star}$, C. Bailloeuil ${ }^{1}$, M. Carles ${ }^{1}$, F. Vera ${ }^{1}$ et I. Sari-Minodier ${ }^{2,3}$ \\ 1 Service médical EDF-CNPE du TRICASTIN, CS 40009, 26131 Saint Paul Trois Châteaux, France. \\ 2 Service de Médecine et Santé au Travail, Pôle de Santé Publique, Assistance Publique-Hôpitaux de Marseille (APHM), Hôpital Timone, \\ 264 rue Saint-Pierre, 13385 Marseille Cedex 5, France. \\ 3 Institut Méditerranéen de Biodiversité et d'Écologie (IMBE UMR CNRS 7263) - équipe Biogénotoxicologie, Santé Humaine et Environne- \\ ment, CNRS, IRD, Univ. Avignon, Aix Marseille Université, Faculté de médecine, 27 Bd Jean Moulin, 13385 Marseille cedex 5, France.
}

Reçu le 7 septembre 2015 - Accepté le 29 juillet 2016

\begin{abstract}
Résumé - La limite réglementaire actuelle de dose au cristallin apparait trop élevée pour prévenir le risque de cataracte radio-induite. La directive 2013/59/Euratom impose un abaissement de cette limite de $150 \mathrm{mSv}$ à $20 \mathrm{mSv}$ par an. Dans cette perspective, EDF a constitué un groupe de travail national destiné à évaluer les doses au cristallin en vue de définir des mesures de protection et de suivi dosimétriques adaptées. L'évaluation dosimétrique d'une partie des activités s'est déroulée dans la centrale nucléaire du Tricastin. Les intervenants ont été équipés d'un dosimètre frontal nanoDot ${ }^{\mathbb{R}}$ pour la dose au cristallin et d'un dosimètre corps entier OSL InLight ${ }^{\circledR}$ pour les quatre opérations de maintenance suivantes : repose de thermocouples sur le couvercle de la cuve; remise en état des triangles du pressuriseur; expertise des joints de la vanne 001VP du circuit de refroidissement à l'arrêt; décontamination des piscines. Pour chaque opération, réalisée deux fois, le ratio moyen dose cristallin sur dose corps entier a été calculé. Si le ratio est supérieur à 1 , l'activité est considérée comme à risque d'exposition significative du cristallin comparativement au corps entier. C'est le cas pour la remise en état des triangles du pressuriseur (ratio moyen égal à 1,37). Cette activité est donc susceptible de nécessiter une surveillance particulière du cristallin, si elle est réalisée fréquemment par les mêmes intervenants.
\end{abstract}

\begin{abstract}
Prevention of radiation-induced cataracts : lowering the regulatory limit dose and evaluation of maintenance activities potentially at risk in nuclear power plants. The current regulatory dose limit to the lens appears to be too high to prevent the risk of radiation-induced cataracts. The Directive 2013/59/Euratom requires a lowering of the limit of $150 \mathrm{mSv}$ to $20 \mathrm{mSv}$ per year. With this in mind, EDF has set up a national working group intended to assess doses to the lens to define appropriate protective measures and dosimetric monitoring. The dosimetric evaluation of some activities took place in the Tricastin nuclear power plant. Workers were equipped with a nanoDot ${ }^{\mathbb{R}}$ front dosimeter for evaluation of the dose to the lens and an OSL InLight ${ }^{\circledR}$ whole-body dosimeter for the following four maintenance operations: installing thermocouples on the lid of the tank; rehabilitation of the pressurizer triangles; expert assessment of the seals of the 001VP valve of the cooling circuit to the stop; and decontamination of the pools. For each operation, performed twice, the lens dose/whole-body dose mean ratio was calculated. If the ratio of an activity is greater than 1, the activity is considered to have a significant risk of exposure of the lens compared with the whole body. This is the case for the rehabilitation of the pressurizer triangles (average ratio equal to 1.37). This activity is likely to require special supervision of the lens, if it is frequently performed by the same workers. The working group concluded that this activity is likely to exceed the future regulatory limit, depending on the frequency of activities significantly exposing the lens for the same worker.
\end{abstract}

Keywords: Lens / dose limit / dosimetry, occupational / cataract / nuclear worker

\section{Introduction}

La cataracte est une opacification du cristallin accompagnée d'une baisse progressive de l'acuité visuelle. La cataracte

^ daloia-raphael@hotmail.fr radio-induite est une complication tardive quasi constante de toute irradiation du cristallin en radiothérapie, dont la sévérité et le délai d'apparition sont fonction de nombreux facteurs tels la dose, son fractionnement et le débit de dose. Un lien est également mis en évidence entre cataracte et exposition aux rayonnements ionisants, pour de faibles doses (Blakely et al., 
2010). Suite aux données scientifiques récentes, la limite actuelle de dose équivalente au cristallin semble trop élevée pour prévenir le risque de cataracte radio-induite. Ainsi, la Commission Internationale de Protection Radiologique (CIPR) recommande, dans sa publication No. 118 (ICRP, 2012), une limite de dose au cristallin de $20 \mathrm{mSv}$ par an, sauf circonstance particulière autorisant $50 \mathrm{mSv}$ sur un an, sous condition que la dose annuelle moyenne sur cinq années consécutives soit inférieure à $20 \mathrm{mSv}$. Cette nouvelle limite a été adoptée dans la directive Euratom du 5 décembre 2013 (Euratom, 2013) et doit être transposée dans le droit français au plus tard le 6 février 2018, passant ainsi d'une limite de dose équivalente au cristallin de $150 \mathrm{mSv}$ à $20 \mathrm{mSv}$, soit une réduction d'un facteur 7,5.

En amont de ce projet d'abaissement drastique de limite de dose, des études ont été entreprises par les professionnels concernés. Ainsi, dans le domaine médical dès 2008, le projet ORAMED (Optimization of RAdiation protection for MEDical staff), associant neuf pays de l'union européenne, a été mis en place avec pour objectifs de déterminer des doses détaillées aux extrémités et au cristallin, lors des procédures de cardiologie et de radiologie interventionnelles (Domienik et al., 2011). Dans le secteur industriel, des études sont également menées pour identifier des postes et activités qui pourraient être concernés par l'abaissement de la limite réglementaire. L'Institut de Radioprotection et de Sécurité Nucléaire (IRSN), dans ses recommandations de bonnes pratiques dans la perspective de l'abaissement de la limite réglementaire pour le cristallin, a identifié les situations suivantes comme pouvant conduire à une exposition significative du cristallin comparativement au reste de l'organisme : le port d'un équipement de protection individuelle (cas du tablier plombé en radiologie interventionnelle par exemple); la géométrie du poste de travail conduisant à ce que la tête se trouve davantage exposée que le reste du corps (opérations en boîte à gants par exemple); le travailleur exposé directement à des rayonnements peu pénétrants (IRSN, 2013).

La présente étude a pour objectif, par des évaluations dosimétriques du cristallin et du corps entier, de déterminer les postes à risque d'exposition du cristallin sur les activités d'Électricité De France (EDF) au sein des Centres Nationaux de Production d'Électricité (CNPE), afin d'ajuster la surveillance dosimétrique et répondre ainsi aux évolutions réglementaires. Elle constitue donc une première approche pour les activités d'EDF permettant d'évaluer si l'exposition du cristallin à certains postes doit nécessiter un suivi dosimétrique spécifique et/ou des modifications de l'ergonomie de ces postes.

\section{Matériels et méthodes}

Suite à la déclaration de la CIPR en faveur de l'abaissement de la dose limite au cristallin, un groupe de travail national, comprenant des représentants EDF, Landauer Europe et Comex nucléaire, a été constitué afin d'évaluer la dosimétrie au cristallin dans plusieurs activités et définir des mesures de protection et de suivi dosimétrique adaptées. Une partie de la présente étude s'est déroulée exclusivement sur le site du CNPE du Tricastin et a été mise en œuvre par le Service Radioprotection et Médical (SRM) d'EDF associé à la société Landauer.
Plusieurs postes potentiellement à risque, pouvant impacter diverses entreprises intervenantes, ont été évalués. Le choix des activités étudiées potentiellement exposantes a été fait sur proposition de la personne compétente en radioprotection, puis validé par le médecin du travail initiateur de l'étude.

Des mesures ont été effectuées avec un spectromètre de terrain tellurure cadmium, dopé au zinc. Les contributions du ${ }^{58} \mathrm{Co},{ }^{60} \mathrm{Co},{ }^{124} \mathrm{Sb}$ au débit de dose en flux direct étaient respectivement de $58,9 \%, 33,4 \%$ et 7,7\%. Le rayonnement étudié est donc composé d'émetteurs bêta et gamma.

L'ensemble des intervenants a été équipé de dosimètres nanoDot ${ }^{\circledR}$ (résultats exprimés en $H_{p}(0,07)$ ) portés à l'aide d'un bandeau frontal pour la mesure de la dose équivalente au cristallin et de dosimètres OSL InLight ${ }^{\circledR}$ (résultats exprimés en $\left.H_{p}(10)\right)$ pour le corps entier, tous deux utilisant une technologie d'oxyde d'aluminium dopé au carbone Le bruit de fond de chaque pastille dosimétrique a été déterminé avant utilisation puis déduit de la mesure finale. Les résultats ont été obtenus par lecture directe sur un lecteur portable micro-star ${ }^{\circledR}$ fourni par la société Landauer.

Comme la réponse énergétique est différente entre les dosimètres OSL InLight ${ }^{\circledR}$ et nanoDot ${ }^{\circledR}$, il a été nécessaire de calculer un coefficient correcteur. Pour déterminer ce dernier, trois dosimètres OSL InLight ${ }^{\circledR}$ et trois dosimètres nanoDot ${ }^{\circledR}$ ont été placés sur un fantôme au niveau du pressuriseur et les différences de réponse énergétique ont été enregistrées. Trois séries de mesures ont été réalisées. Le coefficient correcteur, qui correspond à la moyenne des coefficients correcteurs calculés sur les trois séries de mesures, est égal à 3,05 (Tableau 1).

La campagne de mesures sur le CNPE du Tricastin a porté sur quatre opérations de maintenance à risque, se déroulant lors des arrêts de tranches : 1/ l'expertise vanne 001VP du circuit robinetterie du Réacteur Réfrigération à l'Arrêt (RRA001VP); 2/ la réparation des triangles du Pressuriseur (PZR) ; 3/ la repose de thermocouples sur le couvercle de la cuve du Circuit Primaire du Réacteur (RCP) ; 4/ la décontamination des Piscines de Traitement et de Réfrigération de la cuve (PTR001CU) en fin d'arrêt.

Pour chaque situation étudiée, deux à trois intervenants ont porté les dosimètres sur toute la durée de leur intervention, laquelle a été répartie sur plusieurs jours, en fonction de l'état de l'opération ainsi que des priorités liées directement à l'arrêt de tranche, nécessitant parfois un arrêt temporaire de l'opération en cours. Pour certaines opérations de maintenance, les mesures ont été réalisées uniquement lors de tâches spécifiques. Pour chaque opération, deux évaluations dosimétriques ont été réalisées, identifiées par poste A et poste B. Pour certaines opérations (réparation des triangles et repose de thermocouples), deux opérateurs sont intervenus pour le même poste; leurs résultats dosimétriques ont été alors additionnés. Un taux d'erreur de $20 \%$ est pris en compte pour l'ensemble des évaluations dosimétriques.

Le ratio dose cristallin/dose corps entier a été calculé pour chaque poste étudié. Pour chacune des opérations de maintenance, la moyenne des ratios obtenus pour les postes A et B a été retenue comme critère de jugement : si le ratio est supérieur à 1 , l'activité est considérée comme à risque d'exposition significative du cristallin comparativement au corps entier. 
Tableau 1. Calcul du facteur de correction énergétique sur fantôme placé au niveau du pressuriseur (résultats en mSv). Calculation of the energy correction factor on a phantom placed at the pressurizer (results in $\mathrm{mSv}$ ).

\begin{tabular}{lcccc}
\hline & Série de mesure 1 & Série de mesure 2 & Série de mesure 3 & Total \\
\hline Dosimétrie moyenne $^{1}$ OSL mesurée $(\mathrm{mSv})$ & 1,59 & 1,75 & 1,66 & 5 \\
$\begin{array}{l}\text { Dosimétrie moyenne } \\
\text { nanoDot mesurée }(\mathrm{mSv})\end{array}$ & 0,50 & 0,60 & 0,54 & 1,64 \\
Correction énergétique $^{2}(\mathrm{mSv})$ & 3,18 & 2,91 & 3,07 & 3,05 \\
\hline
\end{tabular}

${ }^{1}$ La dosimétrie moyenne correspond à la moyenne des 3 dosimétries pour chaque série de mesure.

${ }^{2}$ La correction énergétique correspond au rapport de la dosimétrie moyenne OSL mesurée sur la dosimétrie moyenne nanoDot mesurée.

Tableau 2. Caractéristiques des interventions de l'expertise vanneRRA001VP par poste, intervenants, dates, durée. Characteristics of the interventions of the expert assessment of the RRA001VP valve by workstation, workers, date and duration.

\begin{tabular}{|c|c|c|c|c|c|}
\hline \multicolumn{5}{|c|}{ Caractéristiques des interventions } & Total \\
\hline \multirow{4}{*}{ Poste A } & Intervention & No. 1 & No. 2 & & 2 \\
\hline & Intervenant & A1 & A1 & & 1 \\
\hline & Dates & $25 / 07 / 11$ & $27 / 08 / 11$ & & 2 jours \\
\hline & Durée & $12 \mathrm{~h} 45$ & $1 \mathrm{~h} 41$ & & $14 \mathrm{~h} 26$ \\
\hline \multirow{4}{*}{ Poste B } & Intervention & No. 1 & No. 2 & No. 3 & 3 \\
\hline & Intervenant & B1 & B1 & B1 & 1 \\
\hline & Dates & $25 / 07 / 11$ & $27 / 08 / 11$ & $01 / 08 / 11$ & 3 jours \\
\hline & Durée & $7 \mathrm{~h} 46$ & $2 \mathrm{~h} 46$ & $7 h 38$ & $18 \mathrm{~h} 10$ \\
\hline
\end{tabular}

Le responsable de chaque équipe a été au préalable informé des modalités de l'étude. Lors de la campagne de mesures, un technicien du service SRM a suivi le déroulement des activités avec vérification du port des dosimètres. Puis le SRM a relevé et analysé les dosimètres à chaque fin de poste.

Pour chacune des quatre opérations de maintenance, les caractéristiques des interventions étudiées (postes A et B, dates, durées, opérateurs) sont présentées respectivement dans les Tableaux 2 à 5 .

L'activité «d'expertise vanne 001VP» (Tableau 2) porte sur le circuit robinetterie du Réacteur Réfrigération à l'Arrêt (RRA001VP) qui fait partie du circuit carré d'as, ayant pour fonction de maintenir le refroidissement du réacteur lors d'un arrêt de tranche. La «maintenance robinetterie au niveau des vannes RRA001VP» consiste à enlever les servomoteurs, déposer les presses joints, puis à ouvrir des robinets, permettant un accès aux portées de joints pour expertise; c'est lors de cette expertise, exposant potentiellement le cristallin, que les mesures ont été réalisées. Il s'agit d'une activité liée au Programme de Base de Maintenance Préventive (PBMP), dont la fréquence est fournie par un logiciel spécifique.

L'activité de «réparation des triangles du PZR » (Tableau 3) consiste à s'assurer du bon fonctionnement des blocs d'alimentation au niveau du pressuriseur. Cette activité manuelle est réalisée par l'intervenant en montant sur une échelle crinoline pour être au niveau du pressuriseur exposant potentiellement le cristallin. Il s'agit également d'une activité liée au PBMP.

L'activité de « repose de thermocouples » (Tableau 4) consiste en la remise en place des fourreaux d'étanchéité contenant les thermocouples permettant la mesure de la température du cœur; cette opération s'effectue systématiquement lors de chaque arrêt, quel qu'en soit le type.

L'activité de « décontamination des piscines » (Tableau 5) se déroule après la vidange des piscines. Elle consiste dans un premier temps en une décontamination à distance par l'utilisation de canon à mousse. Dans un second temps, il est réalisé une décontamination manuelle avec passage d'une raclette puis de lingettes humides de décontamination et enfin de lingettes sèches. L'intervenant est amené pour cela à descendre dans la piscine en tenue étanche ventilée; c'est sur cette partie de l'activité que les mesures ont été faites. Il s'agit également d'une activité liée à l'arrêt de tranche.

\section{Résultats}

Les caractéristiques des interventions étudiées sont variables en durée et nombre d'intervenants, pour chaque activité de maintenance et chaque poste de travail étudiés (Tableaux 2 à 5). Les opérations se sont déroulées sur 1 à 4 jours, pour des durées totales par opération de 1 h05 à 18 h10. Pour les deux postes A et B, pourtant identiques en termes de travail prescrit, le temps de réalisation peut être identique (cas de la « repose de thermocouples ») jusqu'à plus que doublé (cas de la « décontamination des piscines »).

Le Tableau 6 présente les résultats des deux postes étudiés pour chacune des opérations, après soustraction du bruit de fond et application du facteur de correction énergétique (Tableau 1). Il est à noter une erreur dans le suivi du protocole en ce qui concerne l'opération « expertise vanne RRA001VP», un dosimètre nanoDot ${ }^{\circledR}$ ayant été porté à la poitrine par l'ensemble des intervenants, à la place du dosimètre OSL InLight ${ }^{\circledR}$ prévu. Le deuxième nanoDot ${ }^{\mathbb{R}}$ a bien été porté au niveau frontal, mais les ratios doses équivalentes cristallin/corps entier ne sont donc pas disponibles.

Les postes $\mathrm{A}$ et $\mathrm{B}$ de la «réparation des triangles du $\mathrm{PZR} »$ ainsi que le poste A de la «décontamination des piscines » ont, après soustraction du bruit de fond et application 
Tableau 3. Caractéristiques des interventions de l'activité réparation des triangles du pressuriseur par poste intervenants, dates, durée. Characteristics of the interventions of the repair activity of pressurizer triangles by workstation, workers, date, duration.

\begin{tabular}{ccccccc}
\hline \multicolumn{5}{c}{ Caractéristiques des interventions } & & Total \\
\hline \multirow{4}{*}{ Poste A } & Interventions & 1 & 2 & 3 & 4 & 4 \\
& Intervenant & $\mathrm{A} 1$ & $\mathrm{~A} 1$ & $\mathrm{~A} 1$ & $\mathrm{~A} 1$ & 1 \\
& Dates & $20 / 07 / 11$ & $09 / 08 / 11$ & $10 / 08 / 11$ & $11 / 08 / 11$ & 4 jours \\
& Durée & $1 \mathrm{~h}$ & $1 \mathrm{~h}$ & $3 \mathrm{~h}$ & $8 \mathrm{~h}$ & $13 \mathrm{~h}$ \\
& Intervention & 1 & 2 & 3 & 4 & 4 \\
Poste B & Intervenant & $\mathrm{B} 1$ & $\mathrm{~B} 2$ & $\mathrm{~B} 2$ & $\mathrm{~B} 2$ & 2 \\
& Dates & $20 / 07 / 11$ & $09 / 08 / 11$ & $10 / 08 / 11$ & $11 / 08 / 11$ & 4 jours \\
& Durée & $1 \mathrm{~h}$ & $1 \mathrm{~h}$ & $3 \mathrm{~h}$ & $5 \mathrm{~h}$ & $10 \mathrm{~h}$ \\
\hline
\end{tabular}

Tableau 4. Caractéristiques des interventions de l'activité repose de thermocouples sur couvercle RCP par poste, intervenants, dates, durée.

Characteristics of the interventions of the activity of placing thermocouples on the RCP cover by workstation, workers, date and duration.

\begin{tabular}{ccccc}
\hline \multicolumn{3}{c}{$\begin{array}{c}\text { Repose thermocouple } \\
\text { Caractéristiques des interventions }\end{array}$} & Total \\
\hline \multirow{4}{*}{ Poste A } & Interventions & 1 & 2 & 2 \\
& Intervenant & $\mathrm{A} 1$ & $\mathrm{~A} 2$ & 2 \\
& Dates & $27 / 08 / 11$ & $27 / 08 / 11$ & 1 jour \\
& Durée & $1 \mathrm{~h}$ & $30 \mathrm{~min}$ & $1 \mathrm{~h} 30$ \\
\multirow{4}{*}{ Poste B } & Intervention & 1 & & 1 \\
& Intervenant & $\mathrm{B} 1$ & 1 \\
& Dates & $27 / 08 / 11$ & 1 jour \\
& Durée & $1 \mathrm{~h} 30$ & $1 \mathrm{~h} 30$ \\
\hline
\end{tabular}

Tableau 5. Caractéristiques des interventions de l'activité décontamination des piscines 1PTR001CU par poste intervenants, date, durée. Characteristics of interventions of the activity of pool decontamination (1PTR001CU) by workstation, workers, date and duration.

\begin{tabular}{cccc}
\hline \multicolumn{4}{c}{ Décontamination de 1PTR001CU } \\
Caractéristiques des interventions & Total \\
\hline \multirow{4}{*}{ Poste A } & Interventions & 1 & 1 \\
& Intervenant & A1 & 1 \\
& Dates & $26 / 08 / 11$ & 1 jour \\
& Durée & $1 \mathrm{~h} 05$ & $1 \mathrm{~h} 05$ \\
\multirow{4}{*}{ Poste B } & Intervention & 1 & 1 \\
& Intervenant & B1 & 1 \\
& Dates & $26 / 08 / 11$ & 1 jour \\
& Durée & $2 \mathrm{~h} 30$ & $2 \mathrm{~h} 30$ \\
\hline
\end{tabular}

du facteur de correction, un ratio dose cristallin/dose corps entier supérieur à 1 . Le ratio maximal est de 1,46 pour le poste $\mathrm{A}$ de l'activité « réparation des triangles du PZR » (Tableau 6).

Quant au ratio moyen par activité de maintenance, le seul résultat significatif (ratio nettement supérieur à 1) concerne la « réparation des triangles du PZR », avec un ratio égal à 1,37 (Tableau 6).

\section{Discussion}

Cette étude montre que l'activité de maintenance « réparation des triangles du PZR » est susceptible d'entrainer une exposition significative du cristallin, qui apparait $37 \%$ plus élevée que celle du corps entier. Cependant ce résultat est à considérer avec prudence et n'est pas représentatif de la réalité du terrain, au moment de l'étude. En effet, il convient de rappeler que pour le poste $\mathrm{B}$ de la «réparation des triangles du PZR », de même que pour le poste A de la «repose de thermocouples », ce sont les équivalents de dose mesurés sur deux opérateurs distincts qui ont été additionnés pour le calcul des ratios. On considère donc qu'un intervenant unique a réalisé l'intégralité de l'opération de maintenance, configuration qui est susceptible de se présenter, et qui serait potentiellement la plus à risque d'exposition individuelle.

Le groupe de travail conclut donc que l'activité « réparation de triangles du PZR » doit être considérée comme une activité à risque d'exposition du cristallin eu égard aux recommandations de la CIPR (ICRP, 2012). Il ressort alors la nécessité d'un suivi spécifique de la dose reçue au niveau du cristallin, si cette activité est réalisée de façon fréquente par un même intervenant. Pour assurer ce suivi spécifique et le respect des limites réglementaires, deux propositions émanent du groupe. La première consiste en l'établissement d'un coefficient entre la dose mesurée au niveau de la poitrine et la dose équivalente mesurée au niveau du cristallin. Cette solution se heurte à la nécessité que le spectre rencontré au poste de travail soit homogène et constant; or le type et la quantité de radioéléments varient selon le secteur d'intervention. La deuxième solution est le port de dosimètres adaptés, représentatifs d'une exposition du cristallin. Cette dernière proposition se heurte à la difficulté technique de la mise en place d'un dosimètre avec une ergonomie adaptée à l'activité, tout en restant représentatif de l'exposition du cristallin. Des travaux étaient d'ailleurs en cours au moment de la réalisation de notre étude, tels que ceux conduits par l'IRSN pour la mise au point du dosimètre $\operatorname{DOSIRIS}^{\circledR}$, ou l'actualisation de la norme ISO pour la surveillance du cristallin, de la peau et des extrémités (ISO 15382, 2015).

Quant à la «décontamination des piscines PTR001CU», le ratio moyen (soit 1,09) dépasse très faiblement la valeur 1. Le groupe de travail n'a donc pas retenu cette activité comme étant à risque d'exposition significative du cristallin, en raison de la prise en compte d'un taux d'erreur de $20 \%$ pour les évaluations dosimétriques. Toujours pour la «décontamination des piscines PTR001CU », il est à noter un ratio supérieur à 1 pour le poste $\mathrm{A}$, et non pour le poste $\mathrm{B}$, le ratio du poste $\mathrm{A}$ étant environ $25 \%$ plus élevé que celui du poste $\mathrm{B}$, alors que 
Tableau 6. Ratios dose cristallin/dose corps entier, par poste et ratio moyen pour chaque opération. Lens/whole-body dose ratios per workstation and the average ratio for each operation.

\begin{tabular}{|c|c|c|c|c|c|c|c|c|c|}
\hline \multicolumn{2}{|c|}{ Activité de maintenance } & \multicolumn{2}{|c|}{ Réparation triangles } & \multicolumn{2}{|c|}{ Repose de thermocouple } & \multicolumn{2}{|c|}{ Expertise robinetterie } & \multicolumn{2}{|c|}{ Décontamination piscines } \\
\hline & & Poste A & Poste $\mathrm{B}^{1}$ & Poste $A^{1}$ & Poste B & Poste A & Poste B & Poste A & Poste B \\
\hline \multirow{2}{*}{ Valeur brute (mSv) } & nanoDot $^{(\mathbb{R}}$ & 0,419 & 0,306 & 0,217 & 0,237 & 0,357 & 0,333 & 0,315 & 0,350 \\
\hline & OSL & 0,89 & 0,75 & 0,67 & 0,74 & ND & ND & 0,82 & 1,07 \\
\hline \multirow{2}{*}{ Bruit de fond (mSv) } & $\operatorname{nanoDot}^{\circledR}$ & 0,051 & 0,045 & 0,05 & 0,049 & 0,064 & 0,044 & 0,051 & 0,048 \\
\hline & OSL & 0,12 & 0,13 & 0,14 & 0,11 & ND & $\mathrm{ND}$ & 0,15 & 0,12 \\
\hline \multirow{2}{*}{ Valeur nette $^{3}(\mathrm{mSv})$} & nanoDot ${ }^{\circledR}$ & 0,368 & 0,261 & 0,167 & 0,188 & 0,293 & 0,289 & 0,264 & 0,302 \\
\hline & OSL & 0,77 & 0,62 & 0,53 & 0,63 & ND & ND & 0,67 & 0,95 \\
\hline \multirow{2}{*}{ Valeur corrigée $^{3}(\mathrm{mSv})$} & $\operatorname{nanoDot}^{(\mathbb{2}}$ & 1,12 & 0,80 & 0,51 & 0,57 & 0,89 & 0,88 & 0,81 & 0,92 \\
\hline & OSL & 0,77 & 0,62 & 0,53 & 0,63 & ND & ND & 0,67 & 0,95 \\
\hline $\begin{array}{l}\text { Ratio doses } \\
\text { cristallin/poitrine }\end{array}$ & & 1,46 & 1,28 & 0,96 & 0,91 & ND & ND & 1,20 & 0,97 \\
\hline $\begin{array}{l}\text { Ratio moyen doses } \\
\text { cristallin/poitrine pour } \\
\text { chaque opération }\end{array}$ & & & ,37 & & 0,94 & & ND & & 1,09 \\
\hline
\end{tabular}

\footnotetext{
${ }^{1}$ Deux opérateurs sont intervenus sur le même poste, les équivalents de doses ont été additionnés.

${ }^{2}$ Valeur nette obtenue en soustrayant le bruit de fond à la valeur brute.

${ }^{3}$ Valeur corrigée obtenue en appliquant le facteur de correction énergétique moyen du Tableau 1.

ND : non déterminé.
}

les deux intervenants sont censés effectuer les mêmes tâches. Cela peut s'expliquer par des méthodes et pratiques propres à chaque individu, des gestes supplémentaires en fonction de l'état matériel réel, et une contamination différente lors des interventions.

La principale limite de l'étude est le faible effectif étudié. Il semble en effet difficile de conclure à l'absence de risque d'exposition significative cristallin, sur la base d'une moyenne de deux ratios seulement, alors même que l'un des deux peut être supérieur à 1 , comme dans le cas de la «décontamination des piscines ». Il aurait été intéressant d'étudier un plus grand nombre de postes par activité, pour permettre justement de prendre en compte la variabilité inter-individuelle, tant au niveau des opérateurs que des interventions.

Le choix d'un dosimètre nanoDot ${ }^{\circledR}$ étalonné en $H p(0,07)$ peut être discuté, un dosimètre étalonné en $H p(3)$ étant le dosimètre de référence pour la dosimétrie du cristallin. Toutefois, il n'existait pas au moment de l'étude de document normatif national ou international concernant les conditions de mesure de l'exposition du cristallin (IRSN, 2013). Les dosimètres $H p(0,07)$ sont fréquemment utilisés pour pallier le manque de dosimètres $H p(3)$ (ICRP, 2007). L'utilisation d'un dosimètre étalonné en $H p(0,07)$ se justifie ici, car le spectre présent lors des campagnes de mesures est composé d'émetteurs gamma de hautes énergies comprises entre $511 \mathrm{keV}$ et $1690 \mathrm{keV}$. Or, d'après la Commission Allemande de Radioprotection, le rapport entre doses $H p(0,07)$ et $H p(3)$ est proche de 1 pour des rayonnements d'énergie supérieure à 300 keV (German Commission on Radiological Protection, 2010).

Enfin, il est utilisé, comme critère de jugement, un ratio moyen entre dose cristallin/ dose corps entier supérieur à 1 . Ce critère permet donc de répondre à l'objectif de cette première approche qui était de définir les postes à risque d'exposition significative du cristallin comparativement au corps entier. Pour compléter cette étude, il serait nécessaire de s'intéresser à la fréquence de toutes les activités à risque d'exposition significative du cristallin effectuées sur l'année, pour chaque interve- nant. Ceci permettrait de déterminer si la limite de dose équivalente au cristallin est susceptible d'être dépassée. Au vu de la périodicité des activités de la présente étude (environ une fois par an, lors des arrêts de tranches), de la dose reçue, ainsi que de la diversité des intervenants, il semble peu probable que la future limite réglementaire puisse être dépassée.

\section{Conclusion}

Cette étude a permis d'identifier une activité de maintenance en centrale nucléaire, susceptible de nécessiter une surveillance particulière du cristallin, si elle est réalisée fréquemment par les mêmes intervenants, afin de prévenir le risque de développement d'une cataracte radio-induite.

Cette étude réalisée au sein du CNPE du Tricastin n'est pas exhaustive. D'autres études ont été mises en place afin d'évaluer d'autres activités potentiellement à risque d'exposition significative du cristallin dans le secteur du nucléaire. Ainsi, une étude concernant uniquement le « remplacement des cannes chauffantes » sur différents sites, utilisant un protocole différent, est menée par la société Comex nucléaire, prestataire effectuant cette activité. L'étude du « remplacement des cannes chauffantes » est particulièrement intéressante car cette activité relève d'intervenants spécialisés, qui effectuent cette opération très fréquemment, en se déplaçant sur les différents sites du parc nucléaire (Hebert, 2013). Les premiers résultats ont d'ailleurs mis en avant la nécessité d'une adaptation de l'ergonomie du poste.

D'un point de vue général, cette étude met en évidence l'intérêt, pour les employeurs, les personnes compétentes en radioprotection, les médecins du travail, d'identifier les postes de travail où l'exposition aux rayonnements ionisants est potentiellement à risque pour le cristallin. Cela permet de mettre en place les moyens de protection appropriés et d'optimiser la surveillance. Des études de postes doivent donc être 
entreprises dans tous les domaines, industriels, médicaux ou de la recherche, pour prévenir la cataracte radio-induite.

\section{Références}

Blakely E.A., Kleiman N.J., Neriishi K., Chodick G., Chylack L.T., Cucinotta F.A., Minamoto A., Nakashima E., Kumagami T., Kanamoto T., Kiuchi Y., Chang P., Fujii N., Shore R.E. (2010) Radiation Cataractogenesis : Epidemiology and Biology, Radiat Res 173, 709-717.

Domienik J., Brodecki M., Carinou E., Donadille L., Jankowski J., Koukorava C., Krim S., Nikodemova D., Ruiz-Lopez N., SansMerce M., Struelens L., Vanhavere F. (2011) Extremity and lens doses in interventional radiology and cardiology procedures : first results of the ORAMED project, Radiat Prot Dosim 144, 442447.

Euratom (2013) Directive 2013/59 Euratom du conseil du 5 décembre 2013 fixant les normes de base relatives à la protection sanitaire contre les dangers résultant de l'exposition aux rayonnements ionisants paru au journal officiel de l'union européenne 17.1.2014 L13/1
German Commission on Radiological Protection with Scientific Reasoning (2010) Monitoring the Eye Lens Dose. Dans : 240th meeting of the German Commission on Radiological Protection, February 2, 2010, Bonn.

Hebert M. (2013) Prise en compte du risque d'exposition du cristallin dans les installations ED. Dans : Congrès national de radioprotection SFRP, 11-13 juin 2013 Bordeaux.

ICRP Publication 103 (2007) Recommendations of the International Commission on Radiological Protection, Ann. ICRP 37

ICPR Publication 118 (2012) Statement on tissue Reaction / Early and late Effects of Radiation in Normal Tissues and OrgansThreshold Doses for Tissue Reaction in a Radiation Protection Context, Ann. ICPR 41

IRSN (2013) Rapport PRP-HOM/2013-00010 - Recommandations sur les bonnes pratiques en matière de radioprotection des travailleurs dans la perspective de l'abaissement de la limite réglementaire de dose équivalente pour le cristallin.

ISO 15382 (2015) Radioprotection - Procédures pour la surveillance des doses au cristallin, à la peau et aux extrémités

Cite this article as: R. D’Aloia, C. Bailloeuil, M. Carles, F. Vera, I. Sari-Minodier. Prévention de la cataracte radio-induite : abaissement de la dose limite réglementaire et évaluation d'activités de maintenance potentiellement à risque en centrale nucléaire. Radioprotection 51(4), 265-270 (2016). 